\title{
Use of coupled ion mobility spectrometry-time of flight mass spectrometry to analyze saturated and unsaturated phenylpropanoic acids and chalcones
}

Mwafaq Ibdah ${ }^{1,2}$ and David R Gang ${ }^{1 *}$

\begin{abstract}
Background: In metabolite profiling screens or analyses, where generic separation and analysis conditions are used in efforts to measure as many metabolites as possible, overlapping signals from very similar molecules often make it very difficult if not impossible to separate and identify specific molecules of specific classes. The aim of this study was to evaluate the utility of coupling ion mobility spectrometry to UPLC-TOFMS (UPLC-Q-IMS-TOFMS) as a means to separate and identify saturated and unsaturated phenylpropanoic acids and chalcones, phenylpropanoid-acetate pathway derived compounds that are common in plant extracts.

Results: This approach readily separated most of the unsaturated phenylpropanoid acids (t-cinnamate, $p$-coumarate, caffeate, ferulate) from the corresponding saturated (dihydro-) compounds, and analysis of two dimensional plots of mass/charge ratio values versus ion mobility drift time revealed that the other compounds can indeed be distinguished. However, this approach was less effective for the larger chalcones.

Conclusions: UPLC-Q-IMS-TOFMS is a promising tool to enable the separation, identification and quantification of very similar molecules. Although it has its limitations, as was seen for the chalcones that were not well separated in this investigation, ion mobility spectrometry nevertheless adds an additional level of characterization to large-scale metabolomic screens, which increases the power of such screens without the demand for multiple analyses using very different column chemistries.
\end{abstract}

Keywords: Ion mobility spectrometry, Mass spectrometry, Phenylpropanoic acids, Flavonoids, Chalcone, Dihydrochalcone

\section{Background}

The natural dihydrophenylpropanoid metabolites, such as dihydroferulic acid, dihydrosinapic acid, and dihydrocaffeic acid, have been identified in several plants and accumulate as heartwood-forming constituents [1,2]. These dihydrophenylpropanoids, which contribute to the color, quality, and durability of the woody tissues [2], have either propanol, propionic acid or propanaldehyde side chains, e.g., $p$-dihydrocoumaric acid, dihydroferulic acid, $p$-dihydrocoumaroyl alcohol and dihydroconiferyl

\footnotetext{
*Correspondence: gangd@wsu.edu

'Institute of Biological Chemistry, Washington State University, PO Box 646340, Pullman, WA 99164-6340, USA

Full list of author information is available at the end of the article
}

alcohols. These compounds are of interest to human health because of their multiple biological and pharmacological (antioxidant and radical scavenging) properties $[3,4]$. They differ from their ubiquitous unsaturated counterparts, which are intermediates in the general phenylpropanoid pathway and are similarly widespread in plants, by possessing saturated propanyl side chains.

Chalcones and dihydrochalcones belong to another important class of plant phenolics, the flavonoids. Chalcones are defined by the presence of two aromatic rings linked by a three-carbon bridge that is unsaturated in chalcones and saturated in dihydrochalcones. Chalcones and dihydrochalcones have been found to have a number of important biological activities [5], including anti-
() Chemistry Central

(C) 2014 Ibdah and Gang; licensee Chemistry Central Ltd. This is an Open Access article distributed under the terms of the Creative Commons Attribution License (http://creativecommons.org/licenses/by/4.0), which permits unrestricted use,

distribution, and reproduction in any medium, provided the original work is properly credited. The Creative Commons Public Domain Dedication waiver (http://creativecommons.org/publicdomain/zero/1.0/) applies to the data made available in this article, unless otherwise stated. 
inflammatory [6], antioxidant [7], antiplasmodial [8], anticholinesterase [9] and anticancer properties [10,11], and thus development of means to better detect, identify and measure the levels of such compounds in biological samples is of great importance.

The identification and analysis of such compounds in extracts from complex plant tissues is of great interest for metabolomic analyses and non-targeted phytochemical screens due to their ubiquity in the plant kingdom. However, the precise analysis of phenylpropanoids and flavonoids in plant tissues can be a complex task because of the variety of similar structures present in such molecule classes [12]. As a result, such compounds often co-elute in typical metabolomic or metabolite profiling experiments. Because mass spectrometric analysis is unable to distinguish between isobaric substances and structural information is often lacking due to the absence of standard compounds, which would enable MS/ MS analyses that could lead to better identification and quantification, it is often impossible without an orthogonal analysis technique to identify and quantify specific compounds in these classes, unless complex chromatographic separations utilizing multiple column chemistries is employed, which is undesirable in metabolomicscale investigations.

Ion mobility spectrometry (IMS) coupled inline to UPLC-TOFMS (UPLC-Q-IMS-TOFMS) is a promising and powerful analytical tool for the analysis of the diversity of saturated and unsaturated phenylpropanoidacetate pathway derived compounds in plant extracts $[13,14]$. In the present study, the utility of UPLC-QIMS-TOFMS (using a Waters SYNAPT G2 HDMS instrument) was evaluated for its ability to analyze a series of saturated and unsaturated phenylpropanoic acids and simple chalcones.

\section{Results and discussion}

An Acquity UPLC BEH C18 column was selected for this work due to the separation efficiency, peak shape and signal intensity of the 12 tested compounds, as well as its common use in metabolomic investigations. The combined mixture, consisting of the saturated and unsaturated phenylpropanoic acids and chalcones, eluted within $6 \mathrm{~min}$. In addition to MS analysis, compound elution was monitored by the diode array (220 to $490 \mathrm{~nm}$ ), which showed a broad peak of non-separated compounds for all compound pairs (saturated vs. unsaturated forms). Attempts to optimize compound separation failed to provide baseline separation in the UV chromatograms, using either the BEH C18 or a number of other columns such as CSH (Charged Surface Hybrid) and HSS (High Strength Silica).

Because of the difference in mass between the saturated and unsaturated compounds, and because high resolution MS (i.e., >10,000 full width at half maximum, FWHM) was used, it was possible to differentiate specific compounds in selected ion chromatograms, when standard compounds were available for comparison. However, in the absence of a chemical standard as reference, such as would be expected in bona fide metabolite identification scenarios when metabolites would be analyzed directly from a biological matrix, it would be difficult to identify many of these metabolites based on MS chromatograms and corresponding MS and/or MS/MS spectra alone. Although the application of very high resolution and high mass accuracy mass spectrometry would assist in this characterization, being unable to distinguish regio-isomers, such an approach is still viewed as being inadequate to be able to characterize the compounds of interest in many instances, especially in complex biological samples. To enable better separation and compound verification, IMS was added to the analysis. The drift times in the inline traveling-wave ion mobility spectrometer (TWIMS) were corrected for the massdependent flight time, defined as the time that an ion spent in the TOF analyzer [15].

\section{Analysis of saturated and unsaturated phenylpropanoic acids}

The compounds investigated that were the most simple in terms of structure and functional groups were dihydrocinnamate and trans-cinnamate. As shown in Figure 1, these two compounds overlapped significantly in the UPLC analysis (Figure 1A and C), with retention times at 4.96 and $4.92 \mathrm{~min}$, respectively. Baseline separation of these compounds was not possible under the UPLC conditions tested. However, when IMS was applied to the analysis of this compound pair, the unsaturated compound, $t$-cinnamate displayed a higher mobility (i.e., lower drift time) compared to the saturated dihydrocinnamate, at $1.91 \mathrm{~ms}$ versus $1.98 \mathrm{~ms}$, respectively, (Figure $1 \mathrm{~B}$ and $\mathrm{D}$ ). This difference can be explained by the fact that the double bond of the unsaturated phenylpropanoic acids causes those compounds to be more rigid, and thus potentially more compact, compared to the saturated phenylpropanoic acids, leading to faster movement in the TWIMS, as can be readily seen in the 2-D plot generated by Driftscope (the software used to analyze IMS data in the SYNAPT G2 HDMS system) of drift time versus $m / z$ for the combined mixture (Figure 1E).

The same situation was observed when caffeate ([M$\mathrm{H}]^{-} m / z$ 179.0344) and dihydrocaffeate $\left([\mathrm{M}-\mathrm{H}]^{-} m / z\right.$ 181.0502), with addition of two hydroxyl groups at the meta- and para-positions compared to the $t$-cinnamate/ dihydrocinnamate analogs, were analyzed (see Figure 2). The elution profiles of these two compounds from the UPLC overlapped significantly (Figure 2A and C), as did their drift times, being $1.97 \mathrm{~ms}$ and $2.02 \mathrm{~ms}$, respectively 


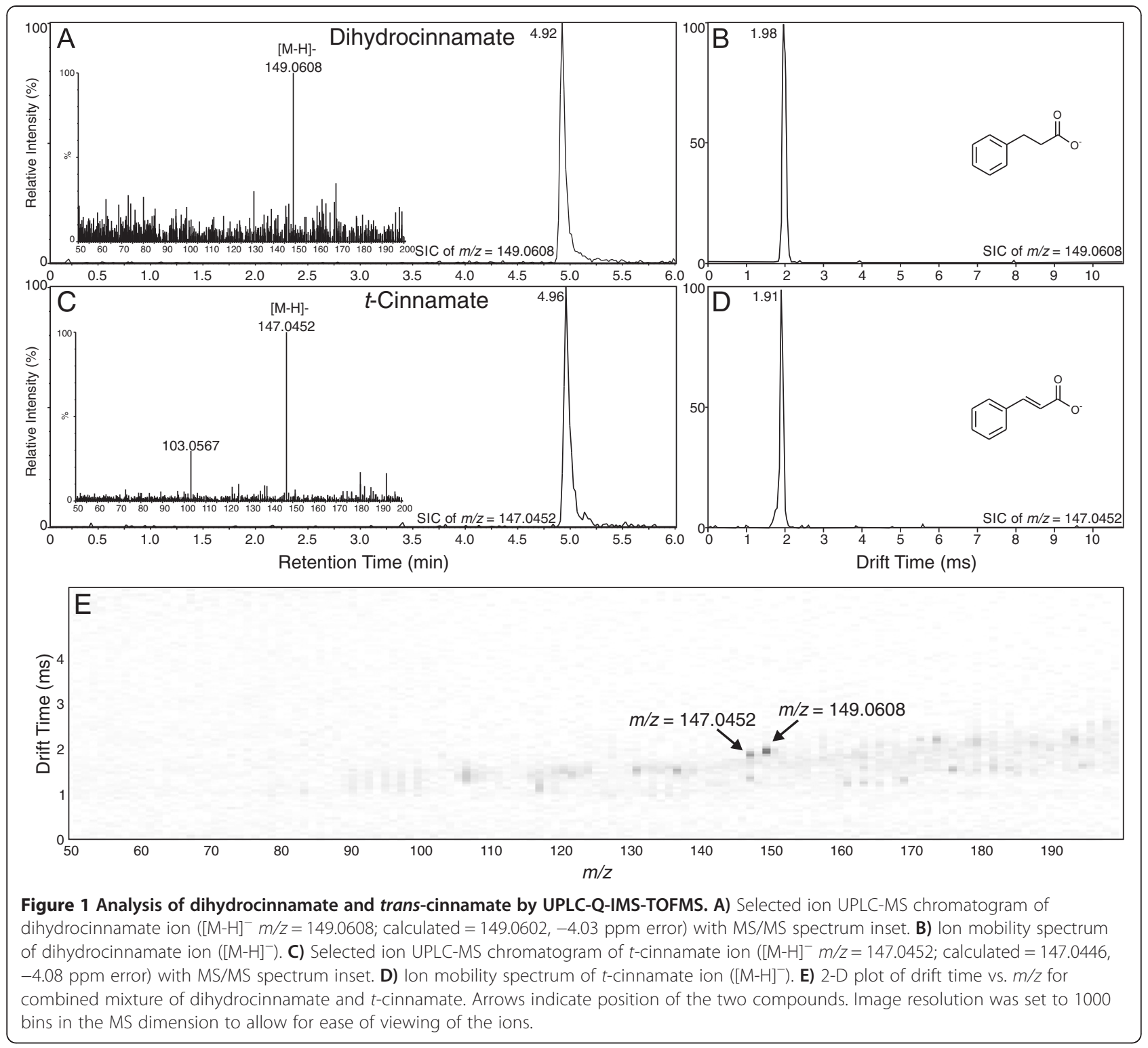

(Figure 2B and D). Thus, analysis by either UPLC-MS or UPLC-IMS alone was not able to separate these compounds. However, as shown in Figure 2E, when UPLC-MS and IMS were applied together in the UPLC-Q-IMSTOFMS system, as shown in the 2-D plot generated by Driftscope of drift time versus $m / z$ for the combined mixture, clear separation of the two compounds is visible, as was seen for the $t$-cinnamate/dihydrocinnamate pair (see Figure 1E).

Continuing with the overall trend of higher drift times based on larger mass, ferulate $\left([\mathrm{M}-\mathrm{H}]^{-} m / z\right.$ 193.0501), the 3-O-methylated derivative of caffeate, and its saturated derivative, dihydroferulate $\left([\mathrm{M}-\mathrm{H}]^{-} \mathrm{m} / z\right.$ 195.0657), displayed the best separation among the hydroxycinnamate pairs (see Figure 3) when analyzed by either UPLC-MS or
UPLC-IMS, with drift times of $2.14 \mathrm{~ms}$ and $2.34 \mathrm{~ms}$, respectively. Being the largest of the hydroxycinnamates, these compounds displayed the lowest mobilities. Being more polar than $t$-cinnamate and dihydrocinnamate but less polar than caffeate/dihydrocaffeate, these compounds had intermediate retention times from the UPLC. As shown in Figure 3E, they were readily distinguishable and identifiable in the 2-D plots in Driftscope.

A more complex compound pair includes $p$-coumarate $\left([\mathrm{M}-\mathrm{H}]^{-} m / z 163.0395\right)$ and $p$-dihydrocoumarate $\left([\mathrm{M}-\mathrm{H}]^{-}\right.$ $m / z$ 165.0545), see Figure 4 . Hydroxylation of the $t$-cinnamate phenyl ring at the para-position leads to $p$-coumarate, which is more polar (earlier elution from the UPLC column) than $t$-cinnamate and ferulate, but less polar than caffeate. As was observed for the $t$-cinnamate/dihydrocinnamate 


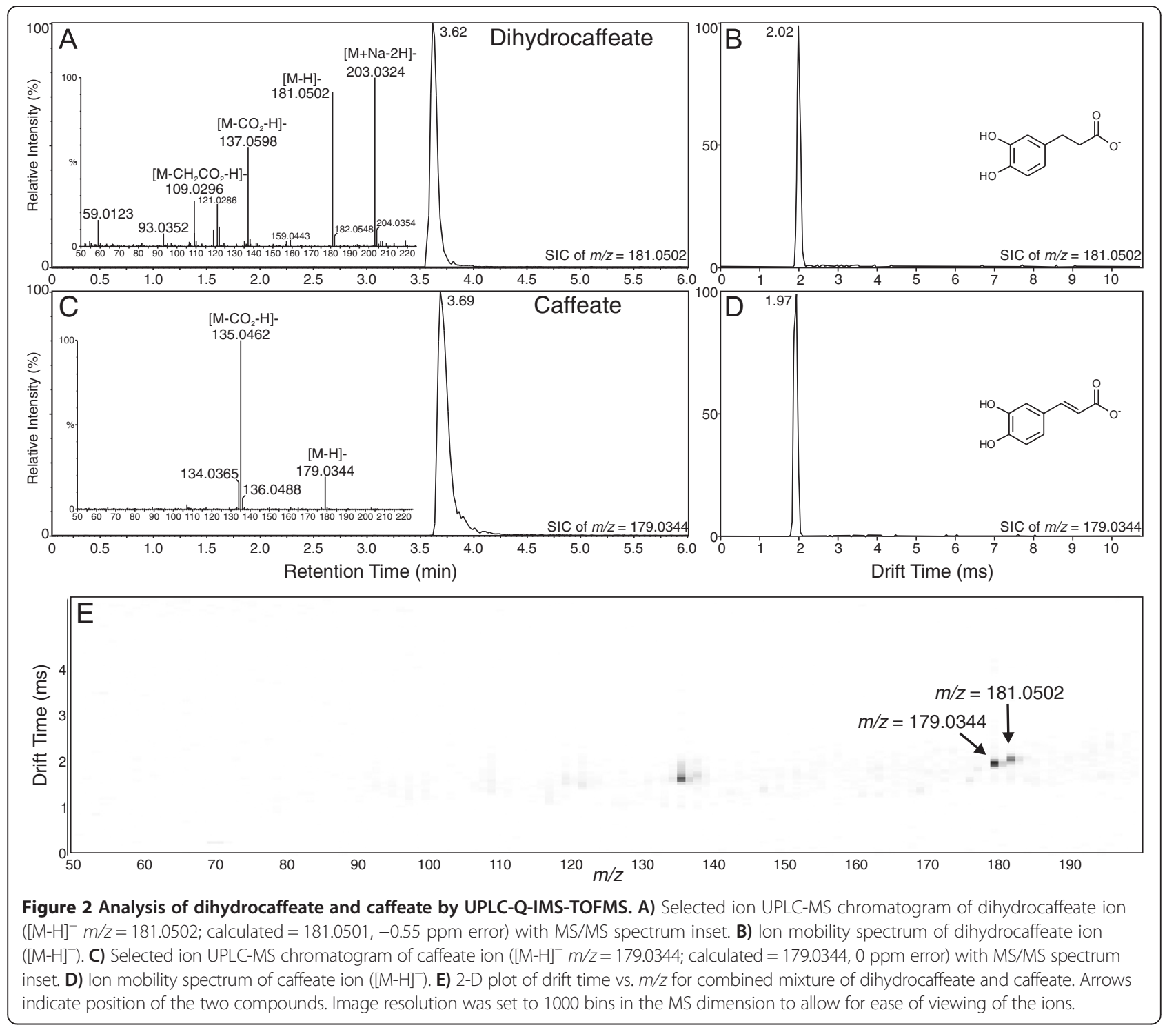

pair, the drift time of $p$-coumarate $(1.86 \mathrm{~ms})$ was shorter than that of $p$-dihydrocoumarate $(2.13 \mathrm{~ms})$. In addition, the drift time of $p$-dihydrocoumarate was longer than that of dihydrocinnamate, its non-hydroxylated but less massive analog. What was surprising was that the drift time of $p$-coumarate $(1.86 \mathrm{~ms})$ was lower than that of $t$-cinnamate (1.91 ms), a presumably more compact molecule, whereas the drift time of $p$-dihydrocoumarate $(2.13 \mathrm{~ms})$ was longer than that of dihydrocaffeate (see above). The cause of these differences may be explained by the nature of the phenolic hydroxyl groups of $p$-coumarate and $p$-dihydrocoumarate, but that is yet to be determined experimentally. Although $p$-coumarate and $p$-dihydrocoumarate were not readily separated in the UPLC-MS analysis, with overlapping chromatograms, they were readily separated, almost to baseline, in the ion mobility analysis (Figure 4B, D and E).

\section{Analysis of saturated and unsaturated chalcones and glycosylated derivatives}

In addition to the hydroxycinnamates described above, two chalcones (naringenin chalcone and naringin chalcone) and their saturated derivatives (phloretin and dihydronaringin chalcone, respectively) were evaluated for their ability to be separated and analyzed by UPLC-MS and IMS under the generic non-targeted metabolomics-based conditions employed in this investigation. As shown in Figure 5, naringenin chalcone $\left([\mathrm{M}-\mathrm{H}]^{-} m / z\right.$ 271.0612) and phloretin $\left([\mathrm{M}-\mathrm{H}]^{-} m / z 273.0876\right.$, the dihydro form of naringenin chalcone) essentially coeluted from the UPLC and had very similar and overlapping drift times in the IMS analysis. The 2-D plot from Driftscope (Figure 5E) shows the overlap in the IMS dimension very clearly. If a low resolution mass spectrometer were to be used in a non-targeted LC- 


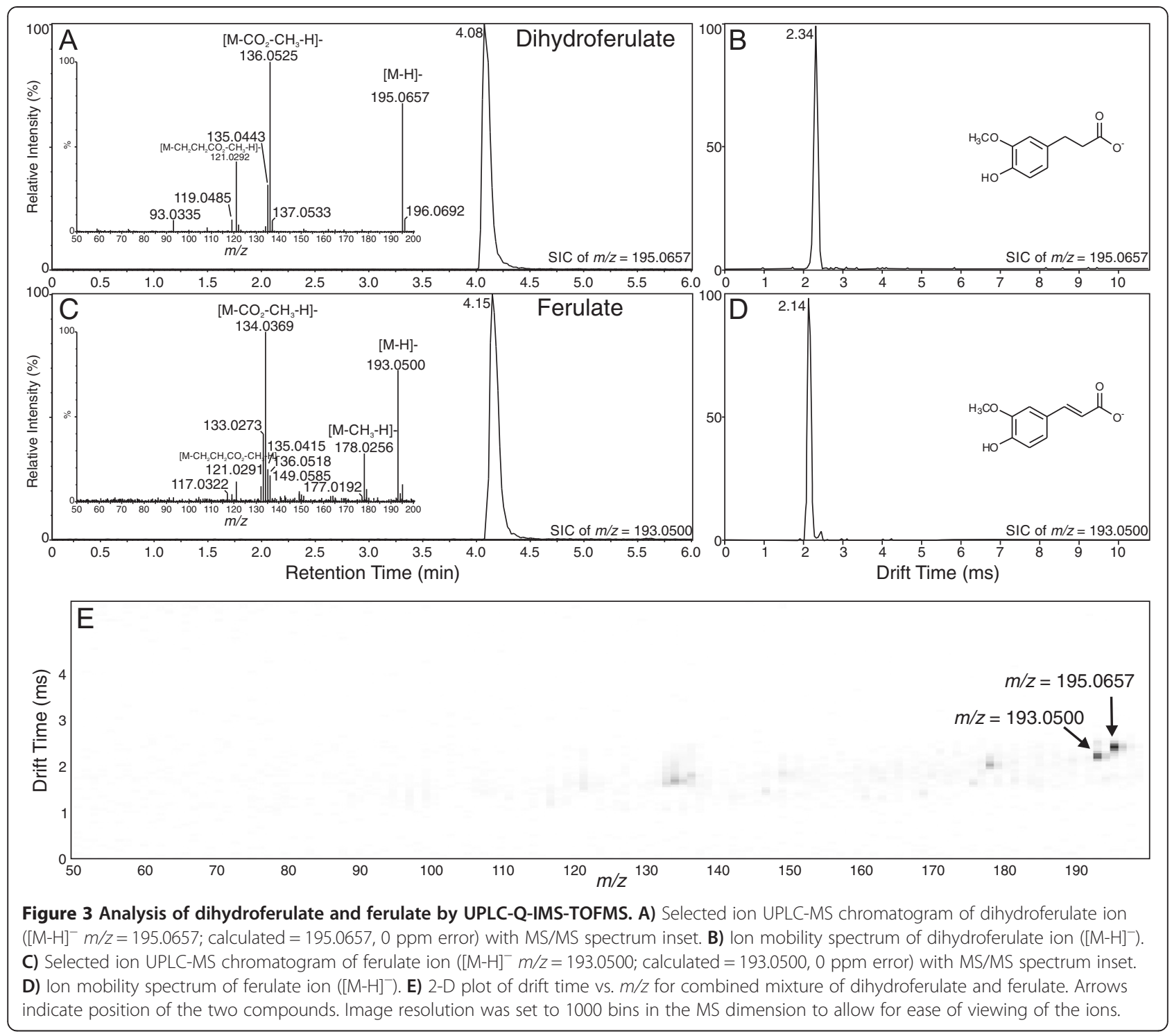

MS/MS-based metabolomics experiment designed to analyze a plant or food extract that was expected to contain these two compounds, and phloretin were present at low levels, it would be impossible to tell it from the $2 \times{ }^{13} \mathrm{C}$ isotopomer of naringenin chalcone using MS analysis alone. Thus, the only way to be sure that both compounds are present is to analyze them by high resolution mass spectrometry or to use a triplequadrupole instrument with an MRM (multireaction monitoring) approach. The latter instrument is typically not used in metabolomic investigations as it is ideally suited instead for targeted metabolite analysis. Thus, even though the data outlined above and other results reported elsewhere [16] showed that ion mobility can be a powerful tool for the analysis of biomolecules, it is not the approach to answer every question.
Naringin chalcone is a glycosylated derivative of naringenin chalcone, with glycosylation occurring on the A ring at position 4 (see Figure 6). As seen for several of the other compounds in this investigation, naringin chalcone $\left([\mathrm{M}-\mathrm{H}]^{-} m / z 579.1907\right)$ and its saturated derivative (dihydronaringin chalcone, $[\mathrm{M}-\mathrm{H}]^{-} \mathrm{m} / z$ 581.2138) shared essentially overlapping elution volumes in the UPLC-MS analysis and very similar behavior in the ion mobility analysis, with drift times of $5.79 \mathrm{~ms}$ for dihydronaringin chalcone and $5.82 \mathrm{~ms}$ for the major conformer of naringin chalcone. A second mobility peak with the same exact mass as the latter compound, with a drift time of $5.22 \mathrm{~ms}$, was clearly visible in the $1 \mathrm{D}$ and $2 \mathrm{D}$ plots (Figure $6 \mathrm{D}$ and E). This peak likely represents a different isomer of naringin chalcone. In any case, the overlapping drift times and UPLC elutions for naringin chalcone and dihydronaringin 


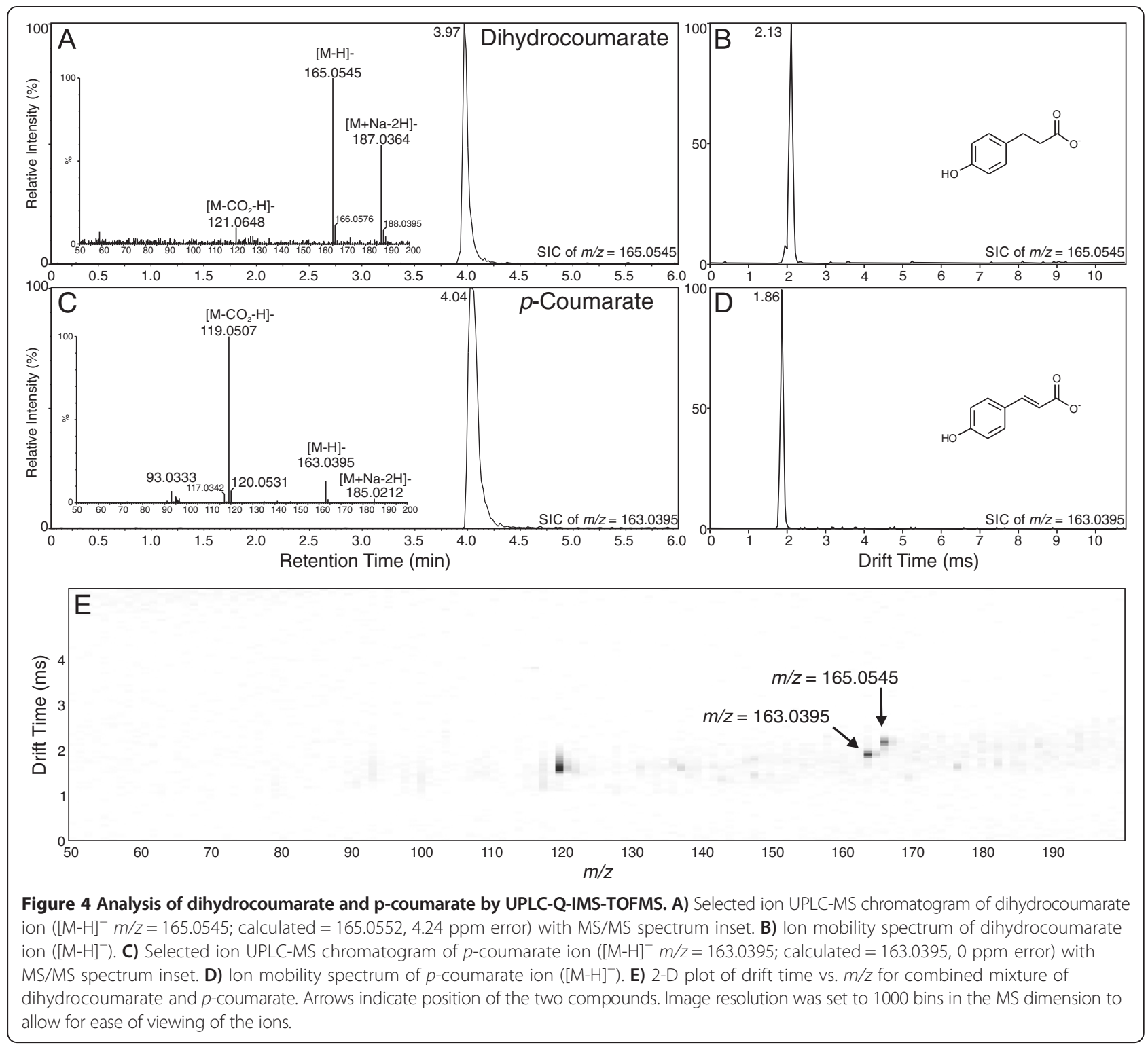

chalcone suggest that, as for the narginenin chalcone/ phloretin story, the only way to tell these two compounds apart in MS-based metabolomics investigations is via high resolution mass spectrometry. Nevertheless, use of IMS in the analysis leads to higher confidence in identification of these compounds, when mobility values are known.

\section{Experimental}

\section{Chemicals}

Standards of several phenylpropanoid acids ( $t$-cinnamate, dihydrocinnamate, $p$-coumarate, $p$-dihydrocoumarate, caffeate, dihydrocaffeate, ferulate, dihydroferulate), chalcones and dihydrochalcones, as well as acetonitrile, ammonium acetate, formic acid, and HPLC grade water, were purchased from Sigma-Aldrich (St. Louis, MO,
USA). The other chalcones used in this investigation were synthesized and purified based on a previously published protocol [17]. All chemicals were dissolved in acetonitrile-water with $0.1 \%(\mathrm{v} / \mathrm{v})$ formic acid, at a final concentration of $100 \mu \mathrm{M}$.

\section{Chromatographic separation}

Chromatography was carried out in an Acquity UPLC system using an Acquity UPLC BEH C18 column of $1.7 \mu \mathrm{m}$ particle size $(2.1 \mathrm{~mm} \times 100 \mathrm{~mm})$, both from Waters (Milford, MA, USA).

UPLC conditions were optimized in order to achieve good chromatographic resolution and sensitivity. Chromatography was carried out at $0.2 \mathrm{~mL} \cdot \mathrm{min}^{-1}$ (total solvent flow rate) and $30^{\circ} \mathrm{C}$ (column temperature). Different 


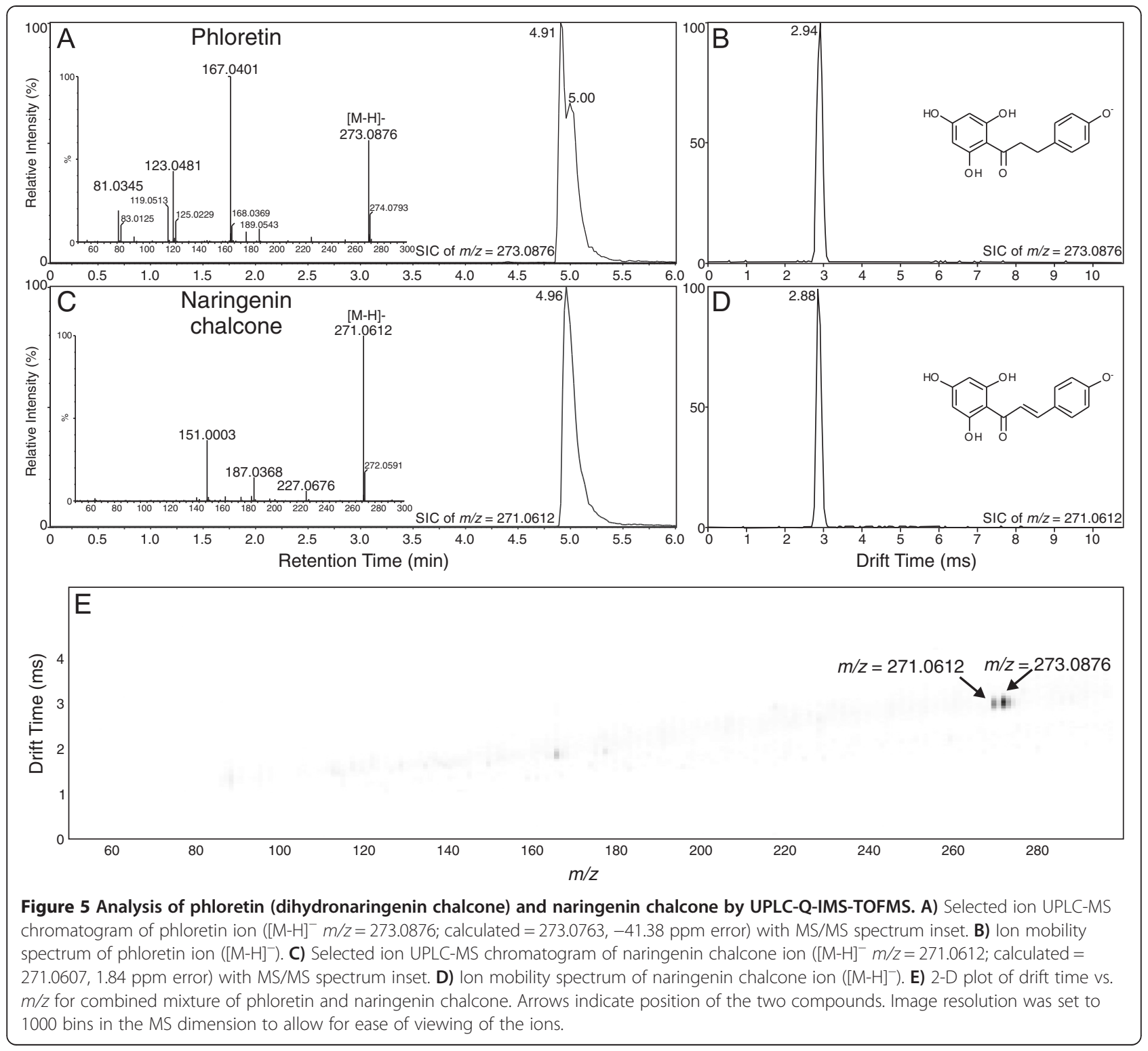

mobile phases and pHs ranging from 2-11 were tested. Finally, the mobile phase that provided the best resolution and which was used for all analyses described consisted of: solvent A, $0.1 \%$ formic acid and $5 \mathrm{mM}$ ammonium formate in water; and solvent B, $100 \%$ acetonitrile and $0.1 \%$ formic acid. The linear gradient system used was $5 \%$ B in A (for $1.71 \mathrm{~min}$ ), 5-65\% B (2.29 min), $65-100 \%$ B (0.5 min), 100\% B (0.5 min), 100-5\% B (2 min). Compound elution was monitored at 200$400 \mathrm{~nm}$ with a Waters UPLC LG500 nm UVNis photodiode array detector. Sample injection volume was $5 \mu \mathrm{L}$.

\section{Mass spectrometry and ion mobility}

All measurements were performed on a SYNAPT ${ }^{\mathrm{mm}}$ G2 HMDS spectrometer (Waters, Milford, MA, USA), which is a hybrid quadrupole time-of-flight (Q-TOF) mass spectrometer with an inline traveling-wave ion mobility spectrometer (TWIMS) between the quadrupole and the TOF. This instrument was connected inline for all experiments with the Acquity system described just above. Thus, the outlet of the LG500 nm UVNis photodiode array detector was transferred directly to the inlet of the SYNAPT ${ }^{\mathrm{mm}}$ G2 HMDS, thereby enabling direct comparison of UPLC-UV/Vis analysis of specific analytes to Q-IMSTOFMS analysis in the SYNAPT G2 HDMS system. Electrospray ionization negative mode (ESI-) was used with a capillary voltage of $3.0 \mathrm{kV}$. The ion source block and nitrogen desolvation gas temperatures were set to 80 and $100^{\circ} \mathrm{C}$, respectively. Cone voltage was $25 \mathrm{~V}$, desolvation gas temperature was $450^{\circ} \mathrm{C}$ and desolvation 


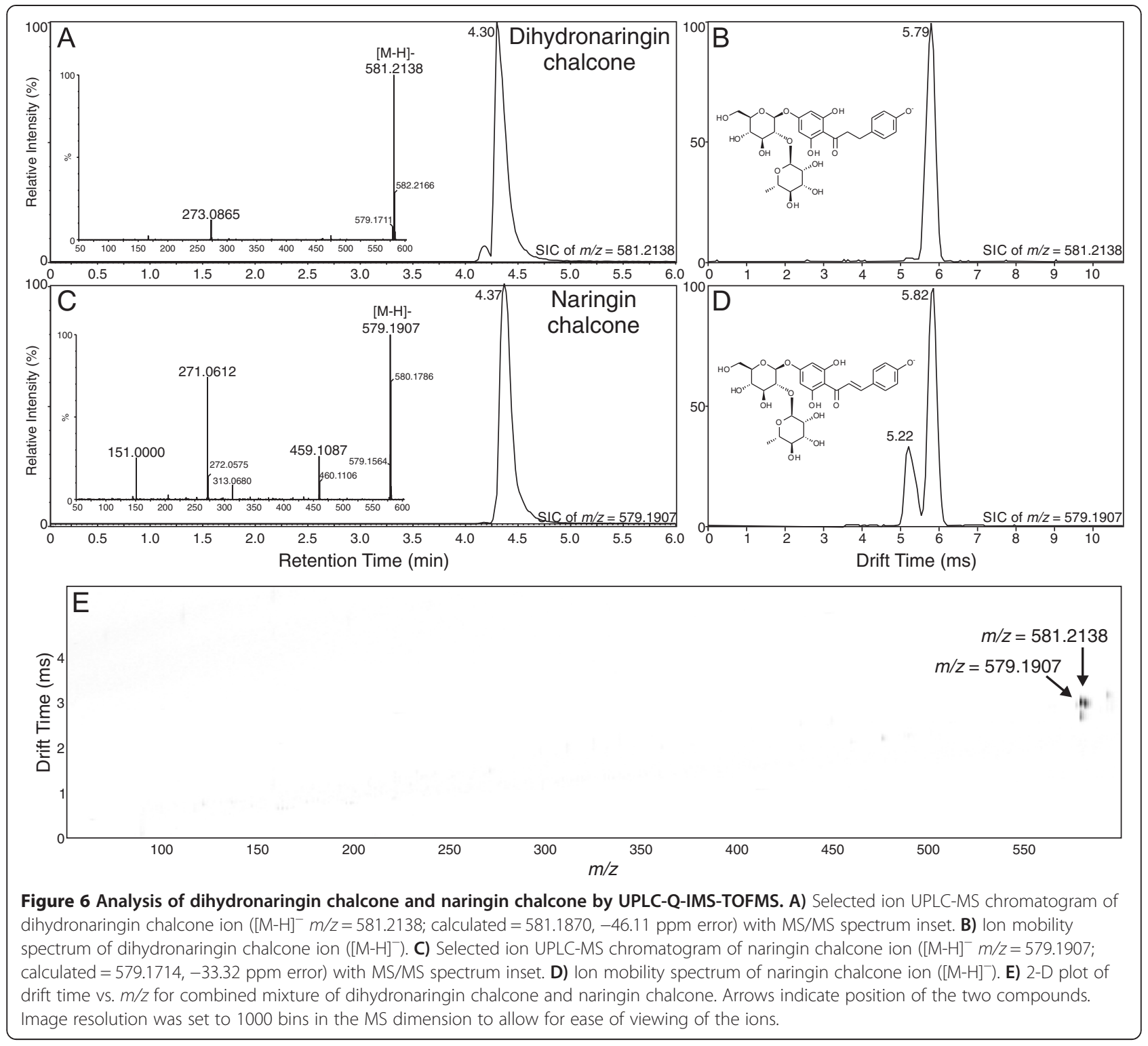

gas flow was $600 \mathrm{~L} \cdot \mathrm{h}^{-1}$. The Mobility T-Wave was operated with a velocity of $250 \mathrm{~m} \cdot \mathrm{s}^{-1}$, the pulse height optimized between 7 and $9 \mathrm{~V}$ and the helium pressure at 3.0 mbar. The TOF-MS spectra were obtained in "V" mode operating at a resolution of $>10,000$ full width at half maximum (FWHM). The mass range acquired was $50-1200 \mathrm{~m} / z$. Data acquisition and processing were carried out using MassLynx (v. 4.1) and Driftscope (v. 2.1) software supplied with the instrument (Waters, Milford, MA, USA).

\section{Conclusions}

In this investigation, ion mobility spectrometry was coupled to UPLC-MS and provided a rapid separation step that enabled a two-dimensional analysis in which chemical noise prevalent in mass spectrometry was significantly reduced (see panel E in Figures 1, 2, 3, 4, 5 and 6). As outlined for the phenylpropanoic acids, IMS also enabled rapid separations of very similar molecules that differed only by the presence or absence of a double bond. There will be cases where it is impossible to resolve such similar molecules by this technique, as was the case for the two chalcones and their dihydrochalcone derivatives. This was likely due to the very small size (collisional cross section area) differences compared to their overall larger masses, although specific conformation preferences for the molecules may have played a role as well. However, as ion mobility resolution and separation power increase, this technique may be able to resolve such compounds or other compounds of 
similar sizes that differ by such small amounts in mass and structure.

\section{Abbreviations}

CSH: Charged surface hybrid; ESI: Electrospray ionization negative mode; FWHM: Full width at half maximum; HSS: High strength silica; IMS: Ion mobility spectrometry; MRM: Multireaction monitoring; Q-TOF: Quadrupole time-of-flight; TWIMS: Traveling-wave ion mobility spectrometer; UPLC-QIMS-TOFMS: ion mobility spectrometry coupled to UPLC-Q-TOFMS.

\section{Competing interests}

The authors declare that they have no competing interests.

\section{Authors' contributions}

Ml performed the experiments and drafted the manuscript. MI and DRG participated in the conception, design of study, revision of the manuscript, and approved the final manuscript

\section{Acknowledgements}

This material is based upon research supported by the National Science Foundation under grant No. MCB-0969010 and the National Institute of Food and Agriculture, U.S. Department of Agriculture, under Agreement No. WNP00765. Mass spectrometric analysis was performed on an instrument acquired through a Major Research Instrumentation grant (DBI-1229749) from the National Science Foundation to DRG.

\section{Author details}

'Institute of Biological Chemistry, Washington State University, PO Box 646340, Pullman, WA 99164-6340, USA. ${ }^{2}$ Agriculture Research Organization, Institute of Vegetable and Field Crops, Newe Ya'ar Research Center, P.O. Box 1021, Ramat Yishay 30095, Israel.

Received: 21 February 2014 Accepted: 9 June 2014

Published: 21 June 2014

\section{References}

1. Leong YW, Harrison LJ, Powell AD: Phenanthrene and other aromatic constituents of Bulbophyllum vaginatum. Phytochemistry 1999, 50:1237-1241.

2. Youn B, Kim SJ, Moinuddin SGA, Lee C, Bedgar DL, Harper AR, Davin LB, Lewis NG, Kang C: Mechanistic and structural studies of apoform, binary, and ternary complexes of the Arabidopsis alkenal double bond reductase At5g16970. J BiolChem 2006, 281:40076-40088.

3. Ordoudi SA, Tsimidou MZ, Vafiadis AP, Bakalbassis EG: Structure-DPPH center dot scavenging activity relationships: parallel study of catecho and guaiacol acid derivatives. J Agric Food Chem 2006, 54:5763-5768.

4. Shimoji Y, Tamura Y, Nakamura Y, Nanda K, Nishidai S, Nishikawa Y, Ishihara N, Uenakai $\mathrm{K}$, Ohigashi $\mathrm{H}$ : Isolation and identification of DPPH radical scavenging compounds in Kurosu (Japanese unpolished rice vinegar). J Agric Food Chem 2002, 50:6501-6503.

5. Di Carlo G, Mascolo N, Izzo AA, Capasso F: Flavonoids: old and new aspects of a class of natural therapeutic drugs. Life Sci 1999, 65:337-353.

6. Vogel S, Barbic M, Jurgenliemk G, Heilmann J: Synthesis, cytotoxicity, anti-oxidative and anti-inflammatory activity of chalcones and influence of a-ring modifications on the pharmacological effect. Eur J Med Chem 2010, 45:2206-2213.

7. Yang WM, Liu JK, Qin XD, Wu WL, Chen ZH: Antioxidant activities of three dihydrochalcone glucosides from leaves of Lithocarpus pachyphyllus. Z Naturforsch 2004, 59:481-484.

8. Portet B, Fabre N, Roumy V, Gornitzka H, Bourdy G, Chevalley S, Sauvain M, Valentin A, Moulis C: Activity-guided isolation of antiplasmodial dihydrochalcones and flavanones from Piper hostmannianum var. berbicense. Phytochemistry 2007, 68:1312-1320

9. Amor ECV, Villasenor IM, Nawaz SA, Hussain MS, Choudhary MI: A dihydrochalcone from Syzygium samarangense with anticholinesterase activity. Philipp J Sci 2005, 134:105-111.

10. Shah A, Khan AM, Qureshi R, Ansari FL, Nazar MF, Shah SS: Redox behavior of anticancer chalcone on a glassy carbon electrode and evaluation of its interaction parameters with DNA. Inte J Mol Sci 2008, 9:1424-1434.

11. Szliszka E, Czuba ZP, Mazur B, Paradysz A, Krol W: Chalcones and dihydrochalcones augment TRAIL-mediated apoptosis in prostate cancer cells. Molecules 2010, 15:5336-5353.
12. Quirantes-Piné R, Lozano-Sánchez J, Herrero M, Ibáñez E, Segura-Carretero A, Fernández-Gutiérrez A: HPLC-ESI-QTOF-MS as a powerful analytical tool for characterising phenolic compounds in olive-leaf extracts. Phytochem Anal 2013, 24(3):213-223.

13. Kanu AB, Dwivedi P, Tam M, Matz L, Hill HH: Ion mobility-mass spectrometry. J Mass Spectrom 2008, 43:1-22.

14. Tang XT, Bruce JE, Hill HH: Design and performance of an atmospheric pressure ion mobility fourier transform ion cyclotron resonance mass spectrometer. Rapid Commun Mass Spectrom 2007, 21:1115-1122.

15. Thalassinos K, Grabenauer M, Slade SE, Hilton GR, Bowers MT, Scrivens JH: Characterization of phosphorylated peptides using traveling wave-based and drift cell ion mobility mass spectrometry. Anal Chem 2009, 81:248-254.

16. Li HL, Bendiak B, Siems WF, Gang DR, Hill HH: Carbohydrate structure characterization by tandem ion mobility mass spectrometry (IMMS)(2). Anal Chem 2013, 85:2760-2769.

17. Gonzalez EA, Nazareno MA, Borsarelli CD: Enthalpy-entropy compensation effect in the chalcone formation from naringin in water-ethanol mixtures. J Chem SocPerkin Trans 2002, 2:2052-2056.

doi:10.1186/1752-153X-8-38

Cite this article as: Ibdah and Gang: Use of coupled ion mobility spectrometry-time of flight mass spectrometry to analyze saturated and unsaturated phenylpropanoic acids and chalcones. Chemistry Central Journal 2014 8:38.

Publish with ChemistryCentral and every
scientist can read your work free of charge
"Open access provides opportunities to our
colleagues in other parts of the globe, by allowing
anyone to view the content free of charge."
W. Jeffery Hurst, The Hershey Company.
- available free of charge to the entire scientific community
- peer reviewed and published immediately upon acceptance
- cited in PubMed and archived on PubMed Central
- yours - you keep the copyright
submit your manuscript here:
http://www.chemistrycentral.com/manuscript/

